Voix et Images

volxetimages

\title{
De l'oeuvre littéraire à la textualité
}

\section{Jean Fisette}

Volume 2, numéro 1, septembre 1976

Fernand Leduc

URI : https://id.erudit.org/iderudit/200031ar

DOI : https://doi.org/10.7202/200031ar

Aller au sommaire du numéro

Éditeur(s)

Les Presses de l'Université du Québec

ISSN

0318-9201 (imprimé)

1705-933X (numérique)

Découvrir la revue

Citer cet article

Fisette, J. (1976). De l'oeuvre littéraire à la textualité. Voix et Images, 2(1),

140-142. https://doi.org/10.7202/200031ar

Ce document est protégé par la loi sur le droit d'auteur. L'utilisation des services d'Érudit (y compris la reproduction) est assujettie à sa politique d'utilisation que vous pouvez consulter en ligne.

https://apropos.erudit.org/fr/usagers/politique-dutilisation/
Cet article est diffusé et préservé par Érudit.

Érudit est un consortium interuniversitaire sans but lucratif composé de l’Université de Montréal, l'Université Laval et l'Université du Québec à Montréal. Il a pour mission la promotion et la valorisation de la recherche. https://www.erudit.org/fr/ 


\section{De I'œuvre littéraire à la textualité}

1976 nous a apporté deux livres de philosophes qui traitent de la question textuelle. Et c'est peut-être un signe des temps que philosophes et littéraires se rencontrent sur un même terrain.

Dans Comment parler de la littérature 1 , Raymond Montpetit esquisse un historique de la "nouvelle critique en France, de G. Poulet à R. Barthes. La problématique instaurée d'entrée est celle de l'herméneutique, posée dans une problématique qui va de Dithley à Ricœur; et il est tout à fait éclairant de revoir la pratique critique d'un Poulet (et: Doubrovsky, Richard, Starobinsky, Rousset...) située ainsi dans une perspective philosophique. Opposée au structuralisme à prétention scientifique, l'herméneutique, réduite à la question littéraire, se marque comme la rencontre de deux consciences (celle de l'auteur, celle du lecteur) dans un même lieuprétexte: l'espace textuel. R. Montpetit poursuit le cheminement du travail sur / dans le texte, depuis cette herméneutique toute empreinte d'humanisme jusqu'à la naissance d'une dialectique du texte produisant de luimême du sens: le texte-machine: «...ça fonctionne..., ça peut se lier..., ça s'écrit, produisant le texte ${ }^{2}$.

Entre ces deux pôles, un déplacement, voire plus, presque une inversion de tous les postulats qui définissent l'objet littéraire, l'étape essentielle étant celle de la disparition de l'auteur, justement en tant que conscience.

Cet historique est posé, non en termes de mouvements, de succession d'écoles, de dates - et c'est là que réside l'intérêt de l'ouvrage - mais dans la perspective des diverses problématiques; car la question essentielle est là, et l'auteur ne cesse de la répéter: qu'est-ce que la littérature? Question peut-être trop globale, mais qui, en vertu de sa hauteur, permet de relier, de produire dans certaines affiliations, les réponses extrêmes apportées depuis vingt ans.

Cette histoire - et R. Montpetit le laisse sentir sans l'expliciter c'est peut-être celle d'un appauvrissement progressif de la conception que l'on se fait de l'œuvre littéraire, un appauvrissement qui serait dû à un examen de plus en plus concentré et trop prolongé sur la même question au point que l'œil n'y trouve plus qu'un reflet de lui-même.

En ce sens, l'itinéraire de $R$. Barthes serait exemplaire; dans Critique et vérité, Barthes posait trois pratiques différentes devant l'œurre littéraire: «la critique, la science et la lecture»; l'œuvre de Barthes aurait suivi ce cheminement dont les productions repères seraient: Michelet, le Degré zéro de l'écriture et $S / Z$. Le point vraiment intéressant dans l'étu- 
de de $R$. Montpetit réside dans la constatation qu'au bout du chemin, R. Barthes doive s'arrêter:

S'arrêter pour être de son temps, pour tenir compte de son moment, la limite ayant trait à l'historique; mais ultimement, s'arrêter pour éviter la maladie (désordre, signifiose), les règles et les limitations que s'impose le discours relevant moins des lois d'une méthode que de celle d'une hygiène: "le désordre du signifiant se retourne en errance hystérique (...) se renverse en subjectivité", alors qu'au contraire, le langage du système étant fermé, clos, transparent et dogmatique, incarne une autre maladie et est "un délire étroitement paranoïaque ". Parler dans l'entre deux sans l'intervalle hystérique / paranoïa et asymbolie / signifiose, les contraintes du discours devant être dictées moins par une logique que pas une pathologique; ainsi se présente la place "critique» de ce discours et l'espace possible de son déplacement ${ }^{3}$.

Voilà un point fixe, celui de l'arrêt et qui prend valeur explicative. Que l'essayiste, citant Barthes d'ailleurs, recourre aux catégories de I'hystérie et de la paranoïa et de la notion fort ambiguë d'hygiène, me semble tout à fait révélateur. C'est qu'à l'intérieur de la problématique relevant proprement de l'écriture, une borne, une clôture a été atteinte. Autant ces catégories empruntées à la psychanalyse peuvent ressembler à un «placage» facile, autant leur incidence appelle un vent neuf qui viendrait des territoires voisins: discours psychanalytique, discours marxiste, etc.

Mais R. Montpetit ne s'aventure pas au-delà de la limite de sa discipline; il se contente de présenter les diverses approches de la littérature qu'il applique au Quatuor d'Alexandrie (L. Durrel) et à Othello (Shakespeare).

R. Montpetit reprend, recense, place en perspective; à ce titre, Comment parler de la littérature constitue un excellent instrument de revision des débats des vingt-cinq dernières années sur la question. Mais un travail qui manque d'imagination.

Et justement, l'Étrangeté du texte de Claude Levesque ${ }^{4}$ apparaît comme la réponse à ce manque. D'inspiration derridienne, cet ouvrage imagine, ouvre des avenues, questionne beaucoup plus qu'il ne répond. C'est que le philosophe Claude Levesque nous propose l'aboutissement d'une réflexion, du travail et de l'expérience d'une carrière. L'entrée en matière donne le ton au livre entier:

Nous ne croyons plus que la pensée voyageuse puisse désormais se séparer de son ombre. L'ombre nous est aussi nécessaire que la lumière. II ne nous paraît plus possible de nous mettre à découvert dans la transparence de la pure lumière, de sortir de l'obscure caverne où ne s'agitent que reflets et mirages, pour voir enfin le Soleil face à face et «saisir la vérité à pleine main " 5 .

Claude Levesque plonge dans le texte de Nietzsche, de Derrida, pour y vivre, s'y perdre dans la mi-clarté, la demi-obscurité. Il y a dans cette atti- 
tude une sorte de générosité que je trouve admirable: celle de l'AVENTURIER qui ne regrette pas l'arrière, le passé. Jamais l'essayiste ne cède à la tentation de s'arrêter sur un sens donné, une valeur sûre; tout est monnaie d'échange pour une monnaie encore plus problématique que la première.

La métaphore (que ce soit le «Puits d'éternité", l' "Abîme de Midi" ou l' "Oeil infini de la montagne», pour retenir quelques points d'ancrage - pistes de lancée chez Nietzsche) ne s'arrête jamais, renvoyant à une image autre et ainsi indéfiniment.

Le fragment final de l'ouvrage ( «la Démesure de la philosophie») se refuse à toute concession ou facilité; il propose un statut essentiel à la pratique philosophique:

Somme toute, c'est comme un corps étranger - qui toujours irrite, - que la philosophie occupe la culture, introduisant en elle un ferment d'insatisfaction chronique, de subversion et de transformation toujours en haleine ${ }^{6}$.

Si l'on reprenait les termes de Barthes, les deux essais ici recensés leur correspondraient avec assez de justesse: un relevé clair et ordonné de la réflexion sur le texte et une errance dans les arcanes de la textualité. La “conscience de l'auteur» dont parlait G. Poulet a cédé la place, la scène, au lecteur dont la pratique relève, non plus simplement de la "conscience" mais de la totalité du sujet: la première topique freudienne (conscient / préconscient / inconscient) est appelée à titre de garant, pour être du même coup, dépassée.

C'est précisément ce jeu, ce glissement constant qui me semble caractéristique du mouvement qu'ont connu les études littéraires des dernières années.

Jean Fisette

Université du Québec à Montréal

1. Raymond Montpetit, Comment parler de la litterature, Montréal, HMH, “Cahiers du Québec n 24, Philosophie», 1976.

2. Ibid., p. 120.

3. Ibid., p. 99. Les citations de Barthes sont tirées respectivement de “Écrivains intellectuels, professeurs ", p. 13 et de Sade, Fourrier, Loyola, Paris, Seuil, «Tel quel $\nsim, 1971$, p. 114.

4. Claude Levesque, l'Étrangeté du texte. Essais sur Nietzsche, Freud, Blanchot et Derrida, Montréal, VLB Éditeur, 1976.

5. Ibid., p. 17.

6. Ibid., p. 242. 